\title{
Erratum to: Use of cellulose nanofibers as a denture immersing solution
}

Wei ZOU ${ }^{1}$, Guang HONG²,3, Yukiko YAMAZAKI ${ }^{1}$, Kazuma TAKASE ${ }^{4}$, Toru OGAWA ${ }^{1}$, Jumpei WASHIO5 , Nobuhiro TAKAHASHI ${ }^{4}$ and Keiichi SASAKI ${ }^{1}$

${ }^{1}$ Division of Advanced Prosthetic Dentistry, Graduate School of Dentistry, Tohoku University, 4-1 Seiryo-machi, Aoba-ku, Sendai 980-8575, Japan

${ }^{2}$ Liaison Center for Innovative Dentistry, Graduate School of Dentistry, Tohoku University, 4-1 Seiryo-machi, Aoba-ku, Sendai 980-8575, Japan

${ }^{3}$ Faculty of Dental Medicine, Airlangga University, Jalan Prof. Dr. Moestopo 47 Surabaya, Indonesia

${ }^{4}$ Department of Prosthetic Dentistry, Graduate School of Biomedical Sciences, Nagasaki University, 1-7-1 Sakamoto, Nagasaki 852-8588, Japan

${ }^{5}$ Division of Oral Ecology and Biochemistry, Graduate School of Dentistry, Tohoku University, 4-1 Seiryo-machi, Aoba-ku, Sendai 980-8575, Japan

Erratum to: Dental Materials Journal 2020; 39(1): 80-88

doi:10.4012/dmj.2018-388 JOI JST.JSTAGE/dmj/2018-388

Authors would like to correct the affiliation of 7 th author.

The author list should be corrected as follows.

\section{Use of cellulose nanofibers as a denture immersing solution}

Wei ZOU ${ }^{1}$, Guang HONG ${ }^{2,3}$, Yukiko YAMAZAKI' ${ }^{1}$ Kazuma TAKASE ${ }^{4}$, Toru OGAWA ${ }^{1}$, Jumpei WASHIO ${ }^{5}$, Nobuhiro TAKAHASHI ${ }^{5}$ and Keiichi SASAKI ${ }^{1}$

\footnotetext{
${ }^{1}$ Division of Advanced Prosthetic Dentistry, Graduate School of Dentistry, Tohoku University, 4-1 Seiryo-machi, Aoba-ku, Sendai 980-8575, Japan

${ }^{2}$ Liaison Center for Innovative Dentistry, Graduate School of Dentistry, Tohoku University, 4-1 Seiryo-machi, Aoba-ku, Sendai 980-8575, Japan

${ }^{3}$ Faculty of Dental Medicine, Airlangga University, Jalan Prof. Dr. Moestopo 47 Surabaya, Indonesia

${ }^{4}$ Department of Prosthetic Dentistry, Graduate School of Biomedical Sciences, Nagasaki University, 1-7-1 Sakamoto, Nagasaki 852-8588, Japan

${ }^{5}$ Division of Oral Ecology and Biochemistry, Graduate School of Dentistry, Tohoku University, 4-1 Seiryo-machi, Aoba-ku, Sendai 980-8575, Japan
} 